clinicas, factores pronósticos y seguimiento a largo plazo. Rev Esp Cardiol. 2015;68:1092-100.

5. Angelini P, Velasco JA, Flamm S. Coronary anomalies. Incidence, pathophysiology and clinical relevance. Circulation. 2002; 105:2449-54.

6. Miranda WR, Oh JK. Constrictive pericarditis: A practical clinical aproach. Prog Cardiovasc Dis. 2017;59:369-79.

7. Cremer PC, Kwon HD. Multimodality imaging of pericardial disease. Curr Cardiol Rep. 2015;17:24.

8. Klein AL, Abbara S, Agler DA, et al. American Society of Echocardiography clinical recommendations for multimodality cardiovascular imaging of patients with pericardial disease: Endorsed by Society for Cardiovascular Magnetic Resonance and Society of cardiovascular computed tomography. J Am Soc Echocardiogr. 2013;26:965-1012.e15.

Zaira Yadira García-López ${ }^{\mathrm{a}, *}$, Moises Jiménez-Santos ${ }^{\mathrm{b}}$, César Antonio Flores-Garcíac , Alejandra Moreno-Vázquez ${ }^{c}$, José Antonio Magaña-Serrano ${ }^{\text {a }}$,

Victor Manuel Prevé-Castro ${ }^{\mathrm{a}}$ y Luis Efrén Santos-Martínez ${ }^{\mathrm{d}}$

a Departamento de Insuficiencia Cardiaca y Trasplante, Unidad Médica de Alta Especialidad, Hospital de Cardiología del Centro Médico Nacional, Siglo XXI, IMSS, Ciudad de México, México
${ }^{\mathrm{b}}$ Departamento de Imagen Cardiovascular, Unidad Médica de Alta Especialidad, Hospital de Cardiología del Centro Médico Nacional, Siglo XXI, IMSS, Ciudad de México, México ' Departamento de Patología, Unidad Médica de Alta Especialidad, Hospital de Cardiología del Centro Médico Nacional, Siglo XXI, IMSS, Ciudad de México, México ${ }^{\mathrm{d}}$ Departamento de Hipertensión Pulmonar y Corazón Derecho, Unidad Médica de Alta Especialidad, Hospital de Cardiología del Centro Médico Nacional, Siglo XXI, IMSS, Ciudad de México, México

* Autor para correspondencia. Avenida Cuauhtémoc No. 330. Colonia Doctores, Delegación Cuauhtémoc, CP 06720, Ciudad de México, México. Correo electrónico:; (ZY GarcíaLópez) Teléfonos:(+52) 56276900; extensión 20212.

Correo electrónico: zayagalz@hotmail.com

(Z.Y. García-López).

https://doi.org/10.1016/j.acmx.2017.06.006 1405-9940/

(c) 2017 Instituto Nacional de Cardiología Ignacio Chávez. Publicado por Masson Doyma México S.A. Este es un artículo Open Access bajo la licencia CC BY-NC-ND (http: / / creativecommons.org/ licenses/by-nc-nd/4.0/).

\section{Síndrome de Wellens: mucho más que una onda $T$}

\section{Wellen's syndrome: much more than a T-wave}

\section{Introducción}

El síndrome de Wellens es una entidad caracterizada electrocardiográficamente por la presencia de ondas $\mathrm{T}$ invertidas 0 bifásicas en precordiales derechas. Fue descrito por Wellens y Zwaan en la década de los 80 y corresponde a un subtipo de angina inestable con alto riesgo de progresar a infarto agudo de miocardio de la cara anterior; estos hallazgos predicen con una alta sensibilidad y especificidad el compromiso de la porción proximal de la arteria descendente anterior, requiriendo una estrategia invasiva temprana con el objetivo de evitar sus complicaciones. A continuación presentamos dos casos de pacientes con síndrome de Wellens con manifestaciones electrocardiográficas diferentes que fueron llevados al laboratorio de hemodinamia para revascularización coronaria percutánea por compromiso de la arteria descendente anterior.

\section{Caso clínico 1}

Hombre de 68 años de edad con antecedentes de diabetes mellitus tipo 2, hipertensión arterial, obesidad y dislipidemia, que acudió al servicio de Urgencias por cuadro de dolor precordial opresivo desencadenado en reposo, de menos de 30 minutos de duración y con clase funcional Killip I. Se realizó electrocardiograma (ECG) tomado en el periodo asintomático que mostró inversión simétrica de ondas $\mathrm{T}$ en precordiales (V1-V6) (fig. 1A) y troponina I con resultado negativo. Se ingresó al laboratorio de hemodinamia bajo la sospecha clínica de un síndrome de Wellens, observando oclusión completa del segmento proximal de la arteria descendente anterior en la coronariografía (fig. 2A); posteriormente se realizó revascularización coronaria percutánea con implante de stent medicado, obteniendo flujo TIMI 3 y excelente resultado angiográfico (fig. 2B).

\section{Caso clínico 2}

Mujer de 72 años con antecedente personal de tabaquismo activo, hipertensión arterial y dislipidemia que acudió al servicio de Urgencias por cuadro clínico de dolor anginoso intermitente de menos de 30 minutos de duración y clase funcional Killip I. Se realizó ECG en el periodo libre de dolor que mostró ondas $\mathrm{T}$ bifásicas en derivadas precordiales (fig. 1B) y troponina I con resultado normal. Se realizó coronariografía evidenciando estenosis crítica de la arteria descendente anterior en su porción proximal (fig. 2C) y revascularización coronaria percutánea con implantación de stent metálico (no medicado), obteniendo flujo TIMI 3 y excelente resultado angiográfico (fig. 2D).

\section{Discusión}

El síndrome de Wellens (SW) es una entidad caracterizada electrocardiográficamente por la presencia de ondas T invertidas o bifásicas en precordiales derechas; las cuales lo categorizan en dos tipos diferentes: el tipo 1, encontrado en el $24 \%$ de los casos, presenta ondas $\mathrm{T}$ bifásicas en las derivadas V2-V3 con una elevación insignificante o inexistente del segmento ST; el tipo 2, es el más frecuentemente encontrado y se caracteriza por la presencia de ondas $\mathrm{T}$ 

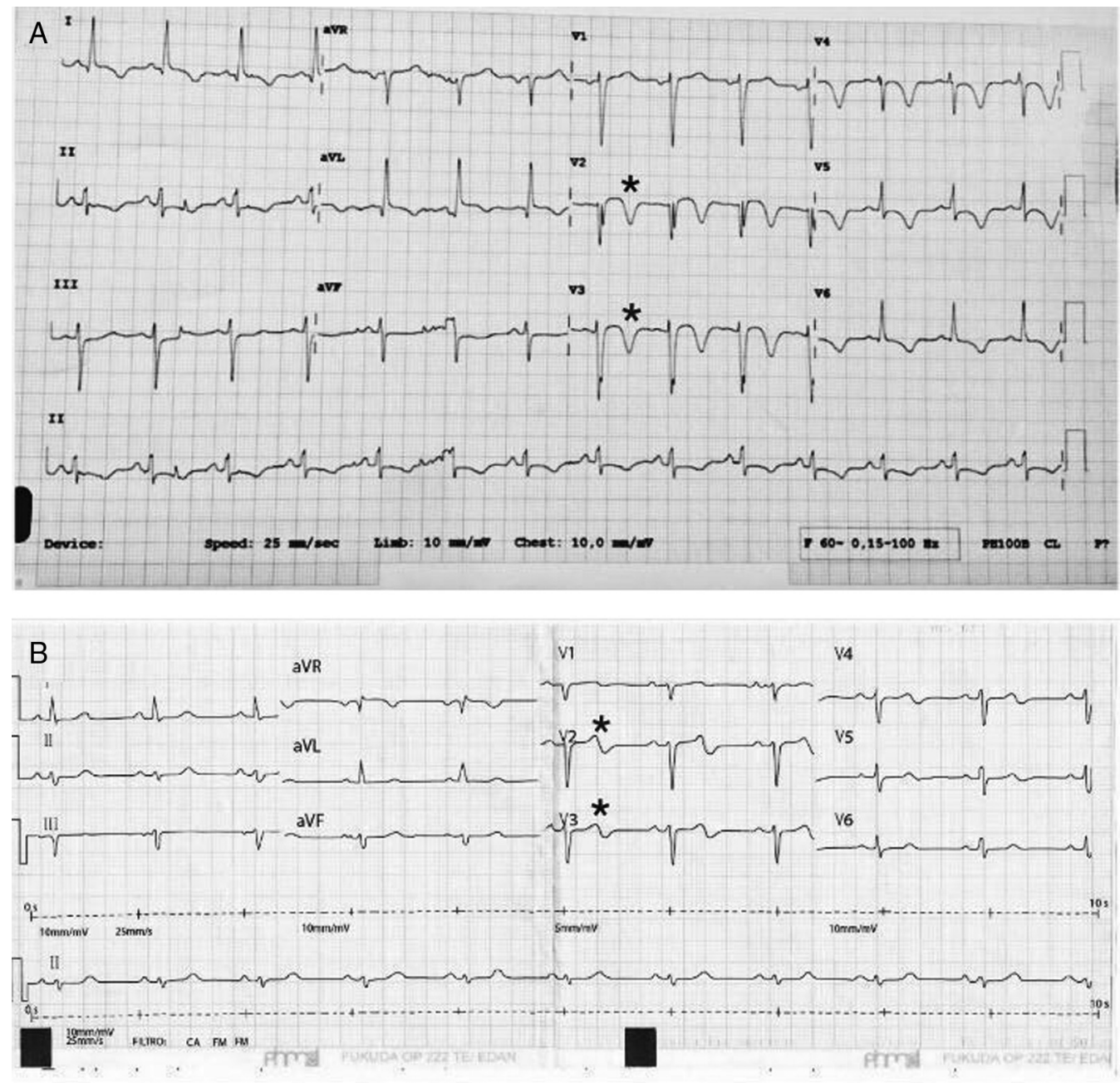

Figura 1 A) Síndrome de Wellens tipo 2: ECG con inversión simétrica de ondas T en derivadas precordiales. B) Síndrome de Wellens tipo 1: presencia de ondas T bifásicas en derivadas del plano horizontal.

invertidas, profundas y simétricas en precordiales derechas, aunque también pueden encontrarse de $\mathrm{V} 1$ a V $5^{1-4}$.

Este síndrome fue descubierto en 1982 por Wellens y Zwaan como un subtipo de angina inestable con alto riesgo de progresar a un infarto agudo de miocardio de la cara anterior; se describió en la literatura como «left anterior descending coronary T-wave syndrome» (síndrome de la arteria descendente anterior), caracterizado por una inversión de la onda $\mathrm{T}$ con leves cambios del segmento $\mathrm{ST}^{5}$. En el primer estudio realizado por Wellens y Zwaan, publicado en 1982 , se estudiaron 145 pacientes de los cuales solo 26 (18\%) mostraron dicho patrón electrocardiográfico, el $75 \%$ de los pacientes que no fueron revascularizados desarrollaron un infarto de la cara anterior del miocardio en los siguientes 23 días $^{5}$; posteriormente en 1989 publicaron un análisis mucho más exhaustivo en donde participaron 180 pacientes, siendo la mayoría hombres, en el cual se evidenció que el $89 \%$ de los pacientes con ondas $T$ invertidas en precordiales derechas tenían una estenosis mayor del $50 \%$ de la arteria descendente anterior (ADA) reportada por coronariografía ${ }^{6}$.

La incidencia del SW es del 10-15\% del total de los síndromes coronarios agudos. Los factores de riesgo están compartidos con los factores de riesgo tradicionales para la enfermedad coronaria tales como: obesidad, hipertensión, diabetes mellitus, tabaquismo, historia familiar de enfermedad coronaria, dislipidemia y sedentarismo ${ }^{3,4,7}$. Los mecanismos asociados a los cambios electrocardiográficos no han sido dilucidados con claridad; se habla de la posibilidad de un miocardio aturdido por reperfusión, debido a la oclusión del flujo de la ADA que se restablece espontáneamente y se cree que otros elementos como el espasmo coronario, la sobrecarga ventricular derecha, la enfermedad microvascular o los trastornos inespecíficos de la repolarización miocárdica puedan estar involucrados en su aparición ${ }^{4}$.

Entre los criterios diagnósticos se destaca la ausencia de ondas $\mathrm{Q}$ patológicas, con una elevación insignificante del segmento ST $(<1 \mathrm{~mm})$, progresión normal de la onda $R$ en las precordiales y enzimas miocárdicas normales o sutilmente elevadas. Está asociado a síntomas de angina inestable; sin embargo, este patrón electrocardiográfico se observa en periodos libres de dolor al momento de la toma del $\mathrm{ECG}^{7,8}$.

Dicho patrón se correlaciona con una estenosis crítica del segmento proximal o medio de la ADA, teniendo la inversión 

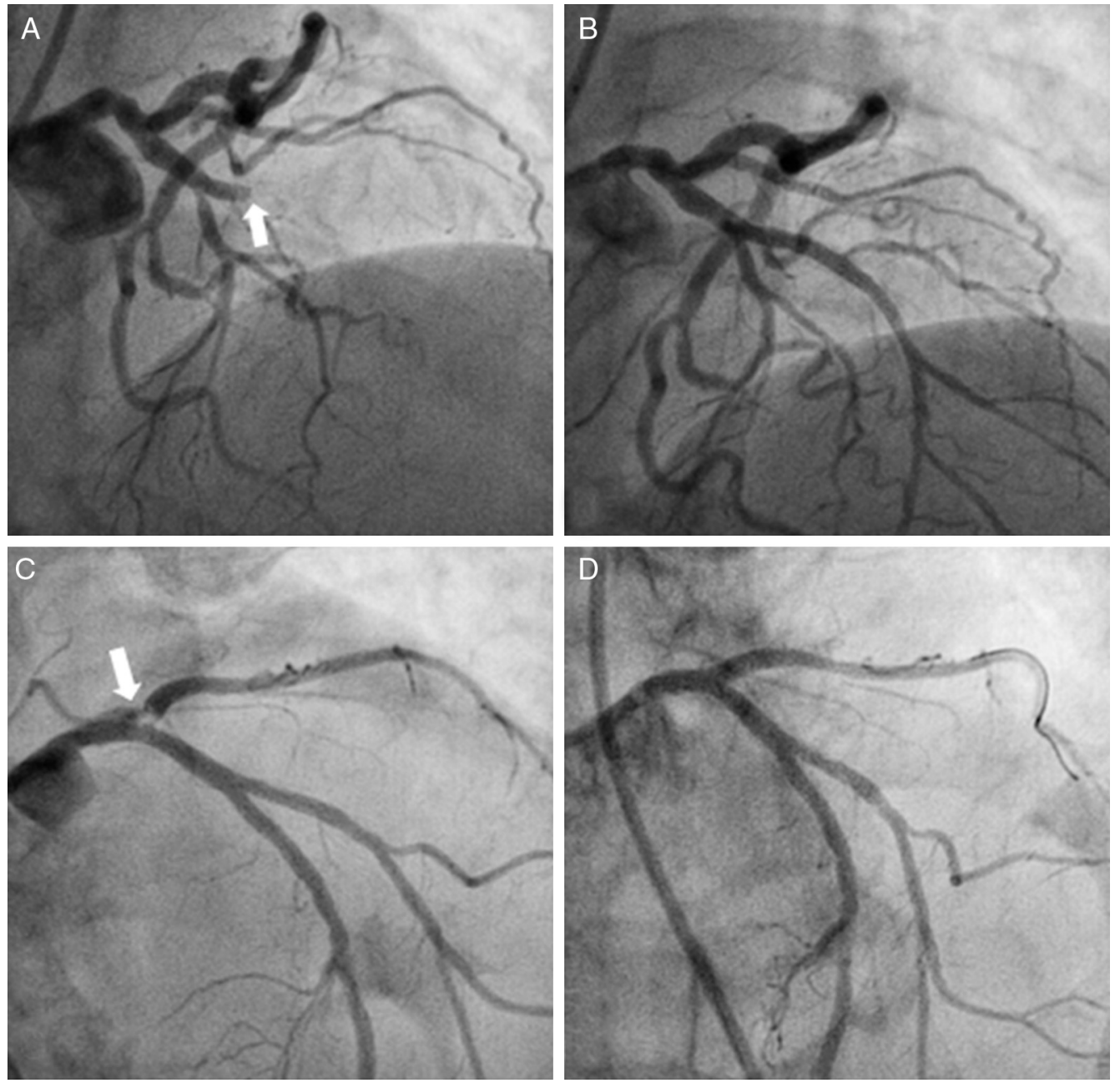

Figura 2 A) Angiografía coronaria evidenciando oclusión completa de la ADA en su segmento proximal. B) Coronariografía posrevascularización coronaria percutánea. C) Estenosis crítica en segmento proximal de la ADA. D) Angiografía coronaria con restauración del flujo después de implantación de stent.

de la onda $\mathrm{T}$ en precordiales derechas una sensibilidad del $69 \%$, especificidad el $89 \%$ y un valor predictivo positivo del $86 \%$ para el hallazgo de una estenosis significativa de esta arteria $^{9,10}$. La realización de una prueba de estrés cardiaca está contraindicada debido a que incrementa la demanda de oxígeno en el miocardio y puede generar desenlaces fatales para el paciente ${ }^{2-4}$.

El SW es de difícil diagnóstico y requiere de una alta sospecha clínica, ya que las ondas $\mathrm{T}$ negativas no solo están presentes en este sino también en otro tipo de patologías, tales como: miocarditis, hipertrofia ventricular izquierda, embolismo pulmonar, síndrome de Wolff-Parkinson-White, efectos digitálicos, eventos isquémicos y patrón de onda T juvenil; además el paciente está asintomático en el momento de la toma del ECG y las enzimas cardiacas son poco relevantes en estos casos, llevando a la interpretación de los cambios electrocardiográficos como inespecíficos ${ }^{3,4}$.

La terapia farmacológica consiste en antiplaquetarios, antitrombóticos, estatinas y betabloqueadores ${ }^{2,6,9}$, aunque cabe resaltar que el manejo con medicamentos es insuficiente para la prevención de la morbilidad y la mortalidad, siendo la intervención coronaria percutánea el tratamiento definitivo, pues si el paciente no es llevado a una revascularización temprana puede presentar disfunción ventricular izquierda, infarto de la cara anterior del miocardio o muerte súbita ${ }^{3}$.

\section{Conclusión}

Es indispensable que el SW sea sospechado y diagnosticado de manera oportuna debido a las particularidades de su presentación y a la gravedad que implica su cuadro clínico en términos de desenlaces. El ECG juega un papel imprescindible en su diagnóstico, por tanto el conocimiento de sus características debe ser una prioridad tanto para los agentes de atención primaria en salud como para el cardiólogo clínico.

\section{Bibliografía}

1. Mai H, Lan X, Lu D, et al. Wellens' Syndrome with a proximal left anterior descending artery occlusion. Clin Case Rep. 2016;4:558-60. 
2. Win Htut Oo SZ, Khalighi K, Kodali A, et al. Omnious T-wave inversions: Wellens' syndrome revisited. J Community Hosp Intern Med Perspect. 2016;6:32011.

3. Ozdemir S, Cimilli Ozturk T, Eyinc Y, et al. Wellens' Syndrome Report of two cases. Turk J Emerg Med. 2016;15:179-81.

4. Carvajal CA, Ardila DJ. Síndrome de Wellens: reconociendo el peligro. Rev Colomb Cardiol. 2015;22:244-8.

5. de Zwaan C, Bär FW, Wellens HJ. Characteristic electrocardiographic pattern indicating a critical stenosis high in left anterior descending coronary artery in patients admitted because of impending myocardial infarction. Am Heart J. 1982;103(4 Pt2):730-6.

6. de Zwaan C, Bär FW, Janssen JH, et al. Angiographic and clinical characteristics of patients with unstable angina showing an ECG pattern indicating critical narrowing of the proximal LAD coronary artery. Am Heart J. 1989;117:657-65.

7. Patel K, Alattar F, Koneru J, et al. ST-Elevation myocardial infarction after pharmacologic persantine stress test in a patient with wellens' syndrome. Case Rep Emerg Med. 2014;2014:530451.

8. Ahmed S, Ratanapo S, Srivali N, et al. Wellens' syndrome and clinical significance of T-wave inversion in anterior precordial leads. Am J Emerg Med. 2013;31:439-40.
9. Jaiswal D, Boudreau D. An incidental case of Wellens' syndrome in a community emergency department. World J Emerg Med. 2016;7:153-6.

10. Ashraf F. Wellens syndrome, a predictor of critical left anterior descending artery stenosis. Postgrad Med J. 2017;93:53.

Jonathan Cardona-Vélez*, Laura Ceballos-Naranjo y Sneider Torres-Soto

Facultad de Medicina, Universidad Pontificia Bolivariana, Medellín, Colombia

*Autor para correspondencia. Cll 78 B No- 72 A-109 Robledo. Medellín, Colombia.

Correo electrónico: jcardonavelez@hotmail.com

(J. Cardona-Vélez).

https://doi.org/10.1016/j.acmx.2017.07.002 1405-9940/

(c) 2017 Instituto Nacional de Cardiología Ignacio Chávez. Publicado por Masson Doyma México S.A. Este es un artículo Open Access bajo la licencia CC BY-NC-ND (http:/ / creativecommons.org/

licenses/by-nc-nd/4.0/).

\section{Cor triatriatum, una causa inusual de evento}

\section{cerebrovascular cardioembólico en adultos. A propósito de un caso}

\section{Cor triatriatum, an unusual cause of a cardioembolic cerebrovascular event in adults. Presentation of a case}

El cor triatriatum (CTTA) es una malformación congénita que corresponde al $0.1-0.4 \%$ de las malformaciones cardiacas congénitas ${ }^{1,2}$. Los primeros informes datan de 1868 y fueron reportados por Church $^{3}$, quien describía una aurícula izquierda (Al) dividida por un septum anormal, pero fue Borst en $1905^{1}$ quien le asignó el nombre de CTTA. La primera corrección quirúrgica se le asigna a Vineberg y Gialloreto en $1956^{1}$. En 1964, Miller et al. describen el diagnóstico angiográfico en la clínica Mayo, y en 1984 Ostman-Smith et al. dan las pautas para el enfoque diagnóstico ecocardiográfico ${ }^{4}$.

No se han reportado diferencias en la incidencia entre sexos ni tampoco una asociación con síndromes genéticos específicos, pero hasta en el $80 \%$ de los casos existe relación con otras malformaciones congénitas, principalmente conexión anómala de las venas pulmonares, foramen ovale permeable y comunicación interauricular tipo ostium secundum ${ }^{1,3,5}$.

Describimos el caso de un paciente con esta enfermedad, mostrando el enfoque diagnóstico realizado, el plan de manejo y las complicaciones que llevaron a un resultado adverso. Se trata de un paciente de sexo masculino, 47 años de edad, con antecedentes de hipotiroidismo e hipertensión pulmonar (HTP) idiopática diagnosticada 2 años atrás, por presentar disnea y deterioro de la clase funcional, apoyados por un cateterismo cardiaco derecho extrainstitucional, que reportó una presión media de la arteria pulmonar de $75 \mathrm{mmHg}$, con prueba de vasorreactividad negativa y una ecocardiografía con signos de HTP e hipertrofia ventricular derecha.

Ingresó al servicio de urgencias por palpitaciones y dolor precordial opresivo e irradiado a miembro superior izquierdo, movimientos masticatorios involuntarios sin pérdida de la conciencia y disnea. Al examen físico presentó: presión arterial de $116 / 80 \mathrm{mmHg}$, frecuencia cardiaca de $98 \mathrm{lpm}$, pulso de $84 \mathrm{ppm}$, saturación de oxigeno del $84 \%$. Ruidos cardiacos arrítmicos, sin soplos, P2 reforzado, ruidos respiratorios disminuidos de forma bilateral. Neurológico sin focalización.

El electrocardiograma mostró fibrilación auricular, frecuencia cardiaca de $140 \mathrm{lpm}$, eje del QRS desviado a la derecha y signos de hipertrofia ventricular derecha (fig. 1a). En la radiografía de tórax: cardiomegalia global, signos de HTP e infiltrados centrales (fig. 1b). Se realizó entonces una ecocardiografía transesofágica (fig. 2) que mostró una Al severamente dilatada, presencia de membrana que divide la Al en dos cámaras, con flujo sistodiastólico hacia la segunda cavidad, más cercana a la válvula mitral por pequeño orificio de $5 \mathrm{~mm}$. Las venas pulmonares drenan a la cavidad superior de la Al (fig. 2a). Además, se observan dos masas en cavidad superior de Al adheridas a la membrana, compatibles con trombos (fig. 2b,c). Orejuela izquierda sin trombos, con velocidades doppler pulsado normal. Aurícula derecha severamente dilatada. Insuficiencia mitral leve y tricúspide moderada, presión sistólica de arteria pulmonar $80 \mathrm{mmHg}$. Ventrículo derecho gravemente dilatado, con función sistólica disminuida. Con estos hallazgos se diagnosticó CTTA sinistrum con presencia de trombos en la membrana y se consideró que la HTP con compromiso de cavidades derechas era secundaria a esta enfermedad y no a una HTP idiopática, como se había considerado en otra institución.

Se definió manejo quirúrgico previa compensación de la fibrilación auricular y falla cardiaca; sin embargo, 3 días 